\title{
DESIGN AND IMPLEMENTATION OF A LINEAR CONTROLLER ON A REDUCED MODEL OF A SMART STRUCTURE
}

\author{
Nader Ghareeb $^{1}$ and Ruediger Schmidt ${ }^{1}$ \\ ${ }^{1}$ RWTH Aachen University \\ Aachen, Germany \\ e-mail: ghareeb, schmidt@iam.rwth-aachen.de
}

Keywords: Finite element modeling, Super element, Classical damping, Lyapunov stability theorem.

\begin{abstract}
Active vibration control of smart structures has gained much attention in the last decades. A simple active control system consists of the mechanical structure where vibration occurs, a sensor to perceive the vibration, an actuator to counteract the influence of disturbances and finally, the controller responsible for the generation of the appropriate control signal. The smart structure used in this work comprises a cantilevered beam with a piezoelement glued to it. A finite element model is initially created and then it is reduced to a super element with a finite number of degrees of freedom. Damping properties are also calculated and added to the reduced model, and the model is verified. Finally, a linear control law based on the Lyapunov stability theorem is used to attenuate the vibrations of the smart beam excited with its first eigenmode. The controller is applied directly to the super element model and after that to the extracted state-space model too. Results from both models are then presented and compared.
\end{abstract}




\section{INTRODUCTION}

Optimizing the weight during the design of structures has the advantage of reducing the manufacturing and operational costs by reducing the amount of raw material used. However, reducing material results in lower stiffness and less damping which make the structure prone to vibration. In addition to reducing the performance of the structural system, vibration can also cause fatigue loads that may lead to failure of the structure itself [1]. One way to overcome this vibration problem is to implement active or smart materials that can be controlled in accordance to the disturbances or oscillations sensed by the structure. Structures incorporating such materials are called smart structures. A smart structure comprises a passive structure and distributed active parts working as sensors and/or actuators. Recent innovations in smart materials and developments in control theory have made it possible to control the dynamics of these structures, and has lead to a big amount of research and development activity in this field [2]. The coupled electromechanical properties of smart materials, which are illustrated in this work as piezoelectric ceramics, make them well-suited for being used as distributed sensors and actuators for controling structural response. As for sensors, mechanically induced deformations can be determined by measuring the induced electrical potential (direct piezoelectric effect); whereas in actuator applications, deformation or strains can be controlled through the introduction of an appropriate electric potential (converse piezoelectric effect) [3]. Active vibration control has initially been applied on ships [4], and then on aircraft and spacecraft [5]. Rapid developments in this field have led to the use of point actuators and sensors to control flexible systems based on the knowledge of elastic mode frequencies and mode shapes at their attachment points [6]. The use of piezoelectric materials as actuators and sensors for noise and vibration control has only been demonstrated extensively over the past thirty years [7]. The studies generally emphasized the capabilities and applications of piezoelements as distributed vibration actuators and sensors by simultaneously controling a finite number of modes of the actual system, like in $[8,9,10]$.

Consequently, the majority of investigations done in this field were carried out either through experiments on the real model as in $[11,12,13]$, or by using 2D or 3D finite element (FE) models of the smart structure as in $[14,15,16]$. However, in the FE work, the damping coefficients were not calculated but rather assumed, which may not reflect the exact performance of the real model.

In the present work, an active linear controller based on Lyapunov stability theorem is designed and implemented to attenuate the vibration of a cantilevered smart beam excited by its first eigenmode. The piezoactuator is modeled, and the relation between voltage and moments at its ends is investigated. A modified FE model of the smart beam based on the first-order shear deformation theory (FOSD) is then created. The damping coefficients are added to the FE model prior to the reduction to a super element (SE) model with a finite number of degree of freedom (DOF). The FE and SE models are validated by performing a modal analysis and comparing the results with the experimental ones. The state-space (SS) model is extracted too. Finally, the Lyapunov controller is integrated into the model to reduce the vibration. The FE package SAMCEF is used for the creation of both the FE and SE models, as well as for the implementation of the controller in the SE model. Consequently, MATLAB/SIMULINK is used for the implementation of the controllers in the SS model. 


\section{MODELING}

In this section, the procedures for modeling a smart structure are examined. The smart structure used in this work is a piezolaminated beam. The same beam model is used later to extract the SE model, derive the SS model, and implement the control strategy. The first step in designing a control system is to build a mathematical model of the structure with all disturbances causing the unwanted vibration. One of the ways to derive the structural analytical model is by using the FE method. The smart beam used consists of a steel beam, a bonding layer and an actuator as seen in Fig. 1.



Figure 1: The smart beam.

\subsection{Actuator modeling}

Using an actuator means implementing an appropriate electric voltage to control the vibration of the smart structure (converse piezoelectric effect). Many FE packages do not offer elements with electrical DOF. Consequently, the voltage applied by the actuator can be represented by two equal moments with opposite directions concentrated at both ends [6]. The relation between actuator moments and actuator voltage can be investigated, so that the moments will then act as the controlling parameters on the smart structure Fig. 2.



Figure 2: The induced stresses from a piezoceramic actuator. 
The procedures to find this relation were already mentioned in $[17,18]$. Based on Fig. 3, the relation between actuator moment and actuator voltage is:

$$
M_{p}=\frac{E_{p} E_{a}\left(t_{p} t_{a}+t_{a}^{2}\right)+E_{p} E_{b}\left(t_{b}^{2}+t_{p} t_{b}+2 t_{a} t_{b}\right)}{E_{p} t_{p}+E_{a} t_{a}+E_{b} t_{b}} \cdot \frac{d_{31} \cdot b}{2} \cdot V
$$

where the indices $p, a$, and $b$ refer to the piazo-actuator, adhesive layer, and steel beam respectively. $t$ is the thickness and $E$ the Young's modulus of each part. The material properties are shown in Tab. 1.
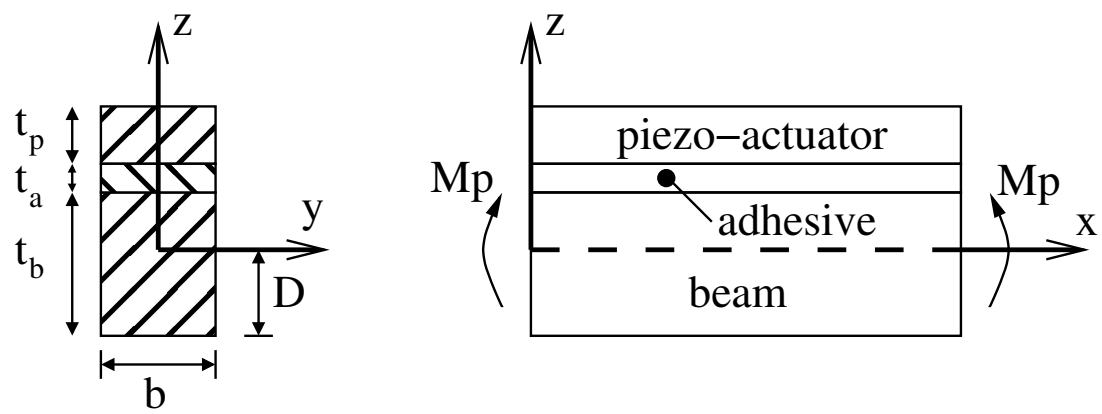

Figure 3: A schematic layout of the composite beam.

Table 1: Parameters of the components of the smart beam.

\begin{tabular}{llll}
\hline & Beam & Bonding & Actuator \\
\hline Material & steel & epoxy resin & $P I C 151$ \\
Thickness $[\mathrm{mm}]$ & 0.5 & 0.036 & 0.25 \\
Density $\left[\mathrm{kg} / \mathrm{m}^{3}\right]$ & 7900 & 1180 & 7800 \\
Young's mod. $[\mathrm{MPa}]$ & 210000 & 3546 & 66667 \\
\hline
\end{tabular}

\subsection{FE modeling}

Finding the best FE model that represents the smart beam used in this work means selecting the optimal element type and size. For this reason, a modal analysis of the real beam is experimentally performed and results of the natural frequencies are compared with those from the FE model where different element types. The FE size which yields the best results will be selected. A detailed geometry of the smart beam is shown in Fig. 4.

The smart beam is created as a unique structure but modeled as a composite shell with three layers. This means, all the three components of the model, i.e. beam, bonding layer and actuator are bonded together without any relative slip among the contact surfaces. Consequently, each layer has its own mechanical properties. To validate the choice of the FE type used (a composite shell element with 8 nodes based on the FOSD), a modal analysis of the FE model is done and the first two eigenfrequencies are read and compared to those from the experiment. This is seen in Tab. 2. As a boundary condition, the far left edge of the smart beam is clamped. 


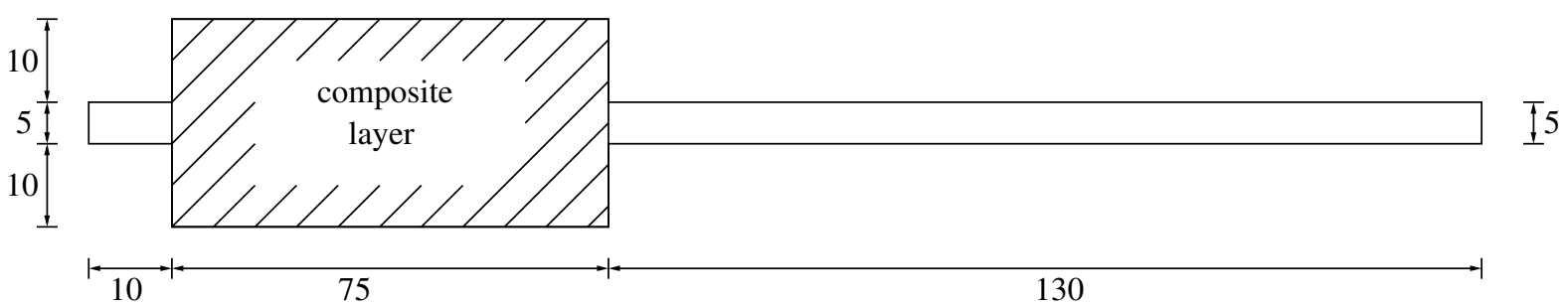

Figure 4: A detailed geometry of the smart beam [dimensions in $\mathrm{mm}$ ].

Table 2: Validation of element-type based on the modal analysis.

\begin{tabular}{lll}
\hline & $F E$ model & Experiment \\
\hline 1st eigenfrequency $[\mathrm{Hz}]$ & 13.81 & 13.26 \\
2nd eigenfrequency $[\mathrm{Hz}]$ & 42.67 & 41.14 \\
\hline
\end{tabular}

Concerning the optimal FE size to be used, it's well known that reducing the FE size will improve the solution accuracy. However, especially in the case of large complex structures, the use of excessively fine elements in the FE model may result in unmanageable simulation periods. From Tab. 3, it is seen that using an element size less than $1 \mathrm{~mm}$ does not make any significant

Table 3: Effect of element size on the eigenfrequency.

\begin{tabular}{lll}
\hline FE size $[\mathrm{mm}]$ & $1 s t$ eigenfreq. $[\mathrm{Hz}]$ & $2 n d$ eigenfreq. $[\mathrm{Hz}]$ \\
\hline 0.25 & 13.80 & 42.66 \\
0.5 & 13.81 & 42.66 \\
1.0 & 13.81 & 42.67 \\
2.5 & 13.83 & 42.71 \\
5 & 13.89 & 42.81 \\
10 & 14.09 & 43.21 \\
\hline
\end{tabular}

change on the values of the 1 st and $2 n d$ eigenfrequencies of the smart beam. This means, it can be regarded as the optimal value for the element size in the FE modeling.

\subsection{SE modeling}

The main virtue of this technique (also called substructure technique) is the ability to perform the analysis of a complete structure by using the results of prior analysis of different regions comprising the whole structure. When a preliminary analysis of the different parts is performed, the computation time and the size of the whole system are reduced. However, all DOF considered useless for the final solution will be condensed and the rest will be retained. This means, the DOF of the whole system will correspond to the retained nodes plus a number of internal deformation modes (dynamic analysis problems). To construct a SE, or in other words to remove the unwanted nodes and DOF from the substructure, many methods that can be used. The 
most popular methods are the "Guyan reduction technique" [19], and the "Component-mode method" which is used in this work.

\subsubsection{The Component-Mode Method}

Also called Craig-Bampton method, it was initially developed by R. Craig and M. Bampton [20]. The idea behind this method is to the basic substructure into a certain number of substructures. The DOF of each substructure are then classified into boundary and internal DOF. The boundary DOF are shared by several substructures, while the internal DOF belong only to the considered substructure.

The behaviour of each substructure is described by the combination of two types of component modes: The constrained modes and the normal vibration modes. The former are determined by assigning a unit displacement to each boundary DOF while all other boundaries DOF are being fixed. The latter correspond to the vibration modes obtained by clamping the structure at its boundary.

It is thus assumed that the behaviour of the substructure in the global system can be represented by superimposing the constrained modes and a small number of normal modes. Hence, by retaining only the low-frequency vibration modes, the substructure's dynamic deformed shape is represented with sufficient accuracy. The method is discussed in details in [21].

Starting from the FE model of the previous subsection, a SE model with a limited number of DOF will be created.

Firstly, the master or retained nodes must be selected. They correspond to the nodes where a boundary condition or a load is applied. The rest of the nodes will be considered as slave or condensed nodes. In the FE model, 5 nodes are considered as retained nodes (Fig. 5).

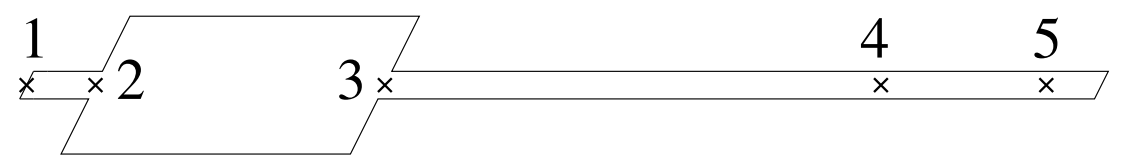

Figure 5: The retained nodes of the SE.

This means:

- Node 1 is used to introduce a boundary condition (clamping constraint).

- Node 2 is used to introduce a load (actuator moment).

- Node 3 is used to introduce a load (actuator moment).

- Node 4 is used to measure the displacement (distance sensor).

- Node 5 is used to measure the tip displacement (distance sensor).

Secondly, 10 modes, which correspond to $97 \%$ of the modal effective mass, are selected. The percentage of modal effective mass for each mode is usually found inside the input file created by any FE software.

To check the validity of the SE created, it has to be compared to the FE model which was already validated before. Abstractly said, the reduced model must have the same characteristics as the original model, except that the number of nodes is reduced, as well as the number of DOF. The eigenfrequencies of the first 2 modes resulting from each model are depicted in Tab. 4. 
Table 4: Eigenfrequencies of the first two modes.

\begin{tabular}{llll}
\hline Mode & SE model $[\mathrm{Hz}]$ & FE model $[\mathrm{Hz}]$ & Experiment $[\mathrm{Hz}]$ \\
\hline 1 & 14.249 & 13.811 & 13.26 \\
2 & 43.414 & 42.673 & 41.14 \\
\hline
\end{tabular}

The physical properties of each model are shown in Tab. 5. It is clear that both models deliver almost the same value of the eigenfrequency for the first two modes. Since the excitation of the beam by its first eigenmode is concerned, furhter readings are not necessary. Compared to the FE model, the SE model has few number of DOF and a very small number of nodes. It consists of a single element and has the advantage that the simulation time becomes short and the controllers are implemented on the SE model itself.

Table 5: Characteristics of the FE and SE models.

\begin{tabular}{lll}
\hline & FE model & SE model \\
\hline Element size $[\mathrm{mm}]$ & 3.07 & \\
Number of elements & 2575 & 1 \\
Number of nodes & 8206 & 5 \\
Number of DOF & 16860 & 40 \\
\hline
\end{tabular}

\section{DAMPING CHARACTERISTICS}

Damping parameters, which are of significant importance in determining the dynamic response of structures, cannot be deduced deterministically from other structural properties or even predicted by using the FE technique. For this reason, recourse must be made to data from experiments conducted on completed structures of similar characteristics. Such data is scarce in general, but it's very valuable for studying the phenomenon and modeling of damping [22]. For simplicity and convenience, the damping is assumed to be viscous and frequency dependent [23]. This linear approach, which was initially introduced by Rayleigh [24], assumes that the damping matrix is a linear combination of the mass and stiffness matrices. Although this idea was suggested for mathematical convenience only, it allows the damping matrix to be diagonalized simultaneously with the mass and stiffness matrices, preserving the simplicity of uncoupled real normal modes as in the undamped case [25].

The relation is

$$
C=\alpha M+\beta K
$$

where $\alpha$ and $\beta$ are real scalars that need to be determined.

The main advantage of this formulation is that the damping matrix will be a diagonal matrix.

This means, the general equation of motion of a damped structure which is given as

$$
[M]\{\ddot{q}\}+[C]\{\dot{q}\}+[K]\{q\}=\{F\}
$$


can be uncoupled to the form

$$
\ddot{u}_{r}+2 \xi_{r} \omega_{r} \dot{u}_{r}+\omega_{r}^{2} u_{r}=\varphi^{T} F=F_{r}
$$

where $q$ is the vector of nodal displacements, $M$ the inertia (mass) matrix, $C$ the damping matrix, $K$ the stiffness matrix, $F$ the column matrix for equivalent nodal forces.

the subindex $r$ means that (Eq. 4) are uncoupled and they correspond to the rth natural frequency of the rth mode that is considered $\left(\omega_{r}\right) . \varphi$ is the vector of mode shapes, $u(t)$ the vector of modal displacements, and $\xi_{r}$ the damping ratio corresponding to the rth mode.

This form of the equation of motion will be used later on to implement the controller on the analytical model of any structure.

Taking the orthogonality of the mode shapes with respect to the mass and stiffness matrices, the modal form of the damping matrix will become

$$
\bar{C}=\varphi^{T} C \varphi=\alpha+\beta \omega_{r}^{2}
$$

this equation can be written in the form

$$
\xi_{r}=\frac{\bar{C}}{\bar{C}_{c}}=\frac{\bar{C}}{2 \omega_{r}}=\frac{\alpha}{2 \omega_{r}}+\beta \frac{\omega_{r}}{2}
$$

with $\bar{C}_{c}$ as the modal critical damping.

From (6), it can be observed that the damping ratio is proportional to the natural frequencies of the system. Roughly speaking, two values for the eigenfrequency $\omega_{r}$ with the corresponding values of $\xi_{r}$ are needed to find out the scalars $\alpha$ and $\beta$ and thus to compute the damping matrix $C$. Relevant are the first few modes for which there is a significant mass participation, i.e. a mass participation which is not less than 95\%. A typical plot of (6) is shown in Fig. 6 [26, 27]. As seen in Fig. 6, the first portion of the curve shows non-linearity (frequency range: $0.1-5 \mathrm{~Hz}$ ), in this case the damping is called "mass proportional damping" where $\alpha$ is much greater than $\beta$. Beyond this portion, the curve is linear (frequency range: $>5 \mathrm{~Hz}$ ). The damping is then called "stiffness proportional damping" and $\beta$ has a big value. To find out $\alpha$ and $\beta$, many methods can be applied like the method of Chowdhury and Dasgupta, or the method of damping from normalised spectra (also known as the half-power bandwidth method) [28]. Both methods are used to find the damping characteristics of the smart beam and the results are depicted in Tab. 6 . In this work, the average values of both methods are used, and the damping coefficients are

Table 6: Results of $\alpha$ and $\beta$ using both methods.

\begin{tabular}{ccc}
\hline Parameter & Chowdhury and Dasgupta & Half-power bandwidth \\
\hline$\alpha$ & 0.02577 & 0.02955 \\
$\beta$ & $9.918 \times 10^{-6}$ & $9.77 \times 10^{-6}$ \\
\hline
\end{tabular}

added to the SE model and to the SS representation. 


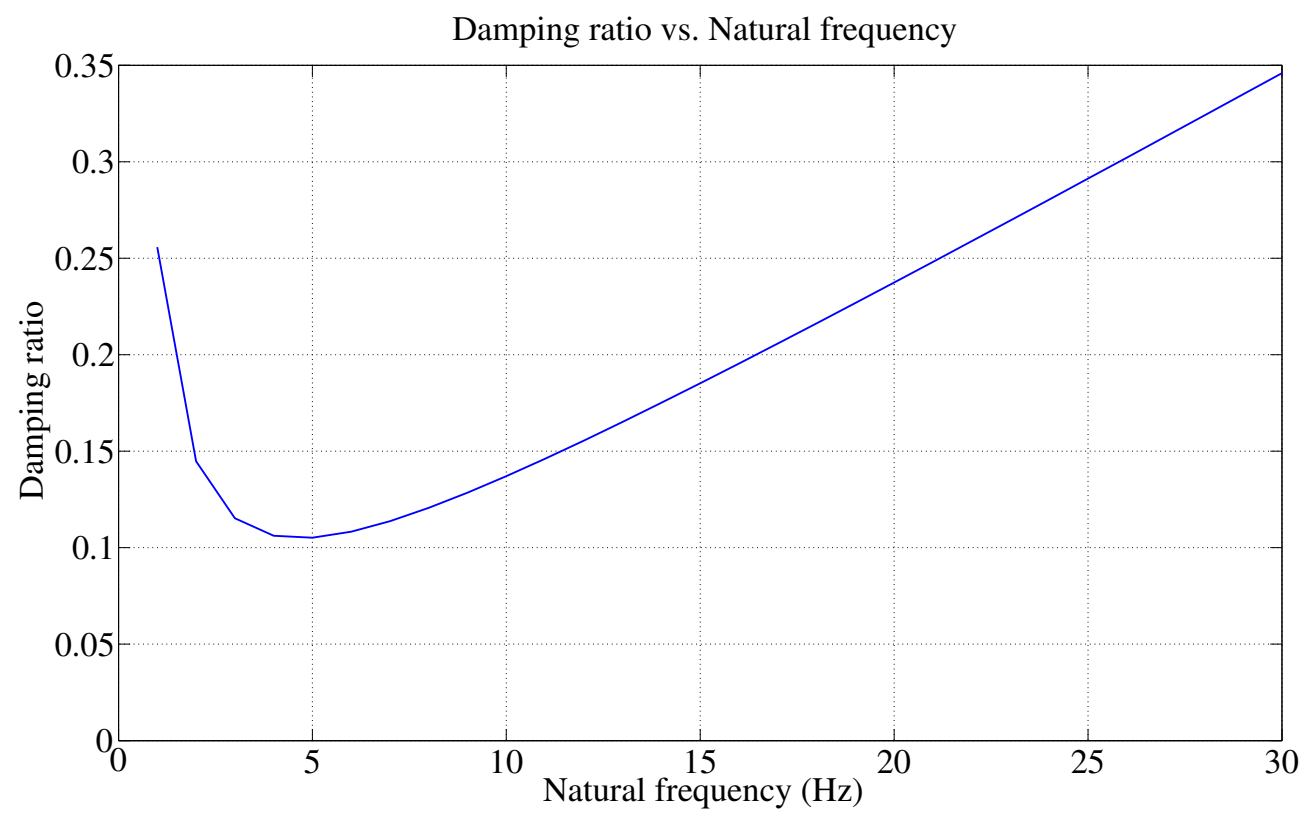

Figure 6: Variation of the damping ratio with natural frequency of a system.

\section{STATE-SPACE REPRESENTATION}

\subsection{Basics of the state-space representation}

The SS representation is used as a second approach to implement the controller in order to validate the results of the SE model. The basic idea of the SS repree is to describe a system of equations in terms of $n$ first-order differential equations. However, the increase in the number of state variables, the number of inputs, or the number of outputs does not increase the complexity of these equations [29].

The state equations have the form:

$$
\begin{aligned}
& \dot{x}=A x+B u \\
& y=C x
\end{aligned}
$$

and the size of the matrices $A, B$ and $C$ depends on the number of states, inputs and outputs of the system. Upon specifying the type and position of the input and output vectors, a FORTRAN code is used to create the SS model of the smart beam. This model is then integrated in MATLAB/SIMULINK to give the dynamic response of the modelled structure under one or several inputs and outputs.

\subsection{Creation and validation of the SS equations for the case of a smart beam}

The objective now is to create the SS representation of the smart structure investigated in this work, and to validate it by carrying out a simple simulation, so that the results can be compared to those from the FE model. At the beginning, the inputs and outputs of the system must be specified. Referring to Fig. 5, a sensor is placed at Node 5 to measure the tip displacement, and the input will be a harmonic force at the same node. Thus, the smart beam will be excited by its first eigenmode. The force $F$ has the form:

$$
F=c \sin \left(\omega_{1} t\right) ; \quad \text { with } c \text { as a constant (amplitude) }
$$


Now, there is a single input and a single output. Since Node 1 is clamped, the number of states is defined as:

$$
2 p=30+10-6=34
$$

There are $30 D O F$ in the system, in addition to 10 vibration modes. Concerning the dimensions of the matrices $A, B, C$ :

$$
\begin{aligned}
\operatorname{dim}(A) & =34 \times 34 \\
\operatorname{dim}(B) & =34 \times 1 \\
\operatorname{dim}(C) & =1 \times 34
\end{aligned}
$$

The SS representation of this smart beam is shown in Fig. 7. The simulation is carried out for 40 seconds while the load is kept active for the first 20 seconds. The resulting curve in Fig. 8 shows that both models had the same value of tip displacement throughout the simulation time. This gives more reliance to the results. However, both models will be used in the coming section for the implementation of the controller.

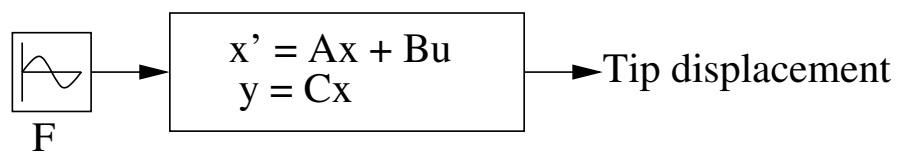

Figure 7: The SS model of the smart beam.

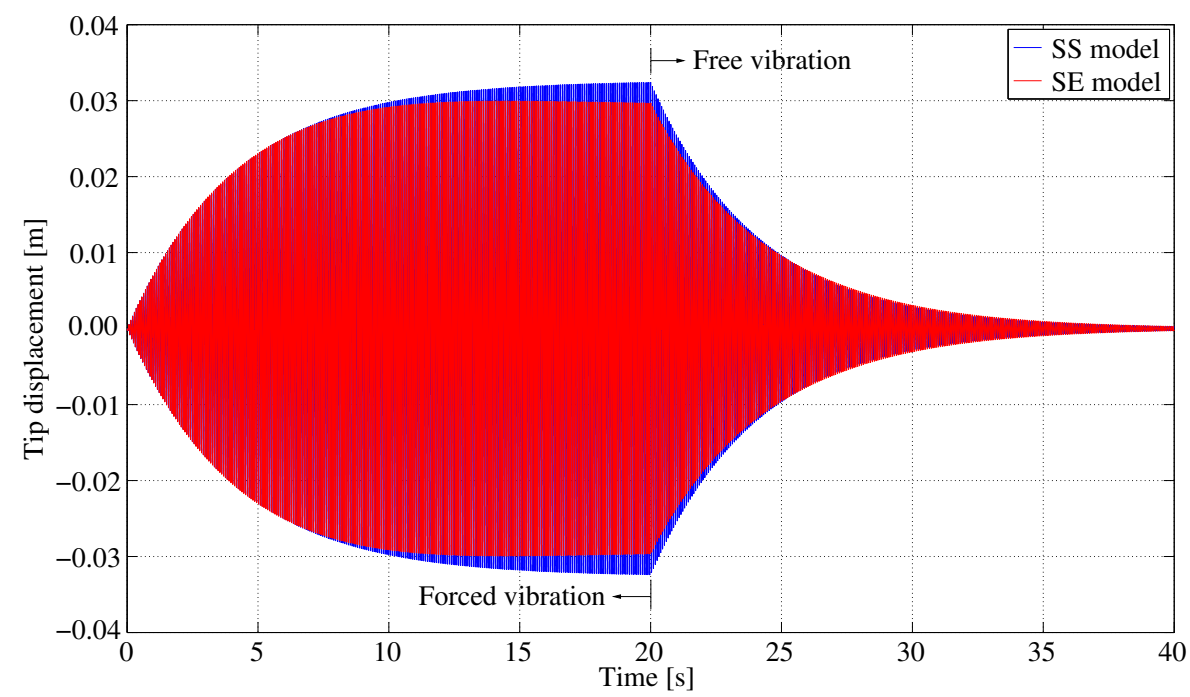

Figure 8: Forced excitation and free vibration of the smart beam.

\section{CONTROLLER DESIGN AND IMPLEMENTATION}

Although there is no general procedure for constructing a Lyapunov function, yet any function can be considered as a candidate if it meets some requirements, i.e., positive definite, equal to zero at the equilibrium state and with its derivative less or equal to zero [30]. Now, the energy equation of a thin Bernoulli-Euler beam which is modelled as a single FE in a one-dimensional 
system with length $h$ and left point coordinate $x_{i}$, is considered as a Lyapunov function candidate (Fig. 9).

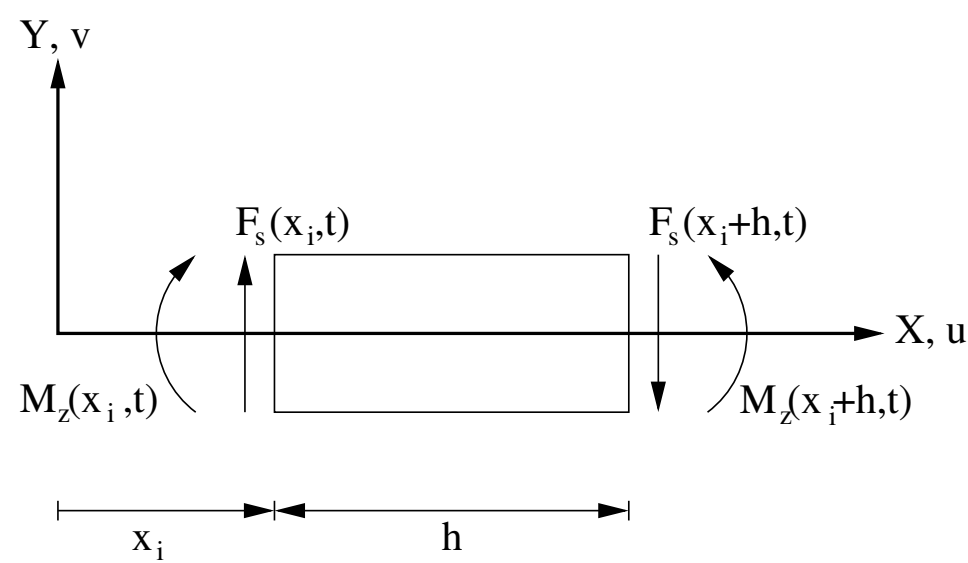

Figure 9: Section of the smart beam where the piezoelement is located.

According to [31], the total energy equation of a beam without any external forces or moments is:

$$
U=\frac{1}{2} \int_{x_{i}}^{x_{i+h}}\left[\rho A\left(\frac{\partial v}{\partial t}\right)^{2}+E I\left(\frac{\partial^{2} v}{\partial x^{2}}\right)^{2}\right] \mathrm{d} x
$$

where $h$ is the length of the beam section, $u$ and $v$ are the displacements in longitudinal and transverse directions, $E$ and $\rho$ are the elastic modulus and density of the beam.

According to Fig. $9, M_{z}$ is the actuator bending moment, $F_{s}$ is the shear force on the beam, and $I_{z}$ is the second moment of inertia of its cross-section about the (bending) z-axis.

The above function is locally positive definite, continuously differentiable and equal to zero at the equilibrium state. Yet, to consider it as a Lyapunov function, the derivative of this function must be smaller or less than zero as well.

Differentiating (11) in time leads to:

$$
\dot{U}=\int_{x_{i}}^{x_{i+h}}\left[\rho A \frac{\partial v}{\partial t} \frac{\partial^{2} v}{\partial t^{2}}+E I \frac{\partial^{2} v}{\partial x^{2}} \frac{\partial}{\partial t}\left(\frac{\partial^{2} v}{\partial x^{2}}\right)\right] \mathrm{d} x
$$

Referring to the relationships for a vibrating beam, which are summarized in any reference about beams like [19], it reveals that:

$$
\begin{aligned}
\rho A \frac{\partial^{2} v}{\partial t^{2}}+E I \frac{\partial^{4} v}{\partial x^{4}} & =0 \\
M_{z} & =E I \frac{\partial^{2} v}{\partial x^{2}} \\
F_{s} & =-E I \frac{\partial^{3} v}{\partial x^{3}}
\end{aligned}
$$

Substituting the derived equations for the bending moment $M_{z}$, shear force $F_{s}$, and assuming no shear after that, the first derivative yields:

$$
\dot{U}=M_{z}\left[\frac{\partial}{\partial t}\left(\frac{\partial v}{\partial x}\right)\right]_{x_{i}}^{x_{i}+h}=M_{z}\left(\dot{v}^{\prime} x_{i}+h-\dot{v}_{x_{i}}\right)
$$


where $\dot{v}^{\prime} x_{i}$ is a rotational velocity at node $x_{i}$.

To ensure that (14) is always smaller or equal to zero, $M_{z}$, the actuator moment, can have the value:

$$
M_{z}=-k\left(\dot{v}^{\prime} x_{i}+h-\dot{v}_{x_{i}}\right)
$$

with $k$ as a positive constant, sometimes called "the proportionality factor". Varying $k$ has a significant effect on the response. Theoretically, the system is stable for any positive value. Nevertheless, larger values of $k$ tend to "overcontrol the structure" since the moment will have a magnitude larger than that required. Consequently, if $k$ is very small, the added moments will be insufficient and this will reduce the damping ratio. Therefore, a trial-and-error procedure is required to select the best value and customize the control to the application [32].

Substituting (15) in (14) yields:

$$
\dot{U}=-k\left(\dot{v}^{\prime} x_{i+h}-\dot{v}_{x_{i}}\right)^{2} \leq 0
$$

and thus, all the requirements to have a Lyapunov function are met. Therefore, (15) can be used as the controller for the smart beam.

To implement this equation on the smart beam, the moments at node 2 and node 3 , which are equal in magnitude but with opposite directions, are calculated as functions of the rotational velocites at both nodes [33]. They have the form:

$$
\begin{aligned}
& M_{2 y}=-k\left(\dot{v}_{2 y}-\dot{v}_{3 y}\right) \\
& M_{3 y}=-k\left(\dot{v}_{3 y}-\dot{v}_{2 y}\right)
\end{aligned}
$$

From the above equations, it is clear that the controller is in fact a connection between the DOF of the nodes composing the SE. To do that in SAMCEF, the nonlinear forces element (FNLI) is used. This element allows the introduction of a list of $n$ general linear or nonlinear internal forces as a function of list of $n D O F$ and their derivatives. The control strategy is defined directly inside the input file without the use of any external programming language, and this is one of the merits of the SE technique.

Coming back to the control law of (17), the controller is stable for any positive value of the constant $k$. Now, the optimal value of the constant $k$ must be found. Since the design of optimal controllers is not the task of this work, the method of trial-and-error is used to find out this optimal value. Best results are got for $k=30$, and the corresponding curve of tip displacement vs. time of the smart beam is illustrated in Fig. 10. In the FFT spectrum diagram (Fig. 11), the effect of the controller on the amplitude of the resonance at the natural frequency is shown as well.

As stated before, the SS representation of the smart beam is derived in order to validate the results from the SE model. To do that, the inputs and the outputs are designated in order to find out the matrices $A, B$ and $C$ of (7). To implement the controller in the SS model, two steps are performed. In step one, the only input to the system is the forced excitation until the magnitude of vibration does not change anymore, i.e., up to $t=20 \mathrm{~s}$, and the output consists of the tip displacement, as well as the state vectors exactly at $t=20 \mathrm{~s}$. The diagram is shown in Fig. 12 .

These state vectors are then fed in as initial conditions in the second step. This time, the input comprises both actuator moments at both ends of the actuator, and the output embraces the tip displacement at node 5 , and the velocities at the node 2 and node 3 . 


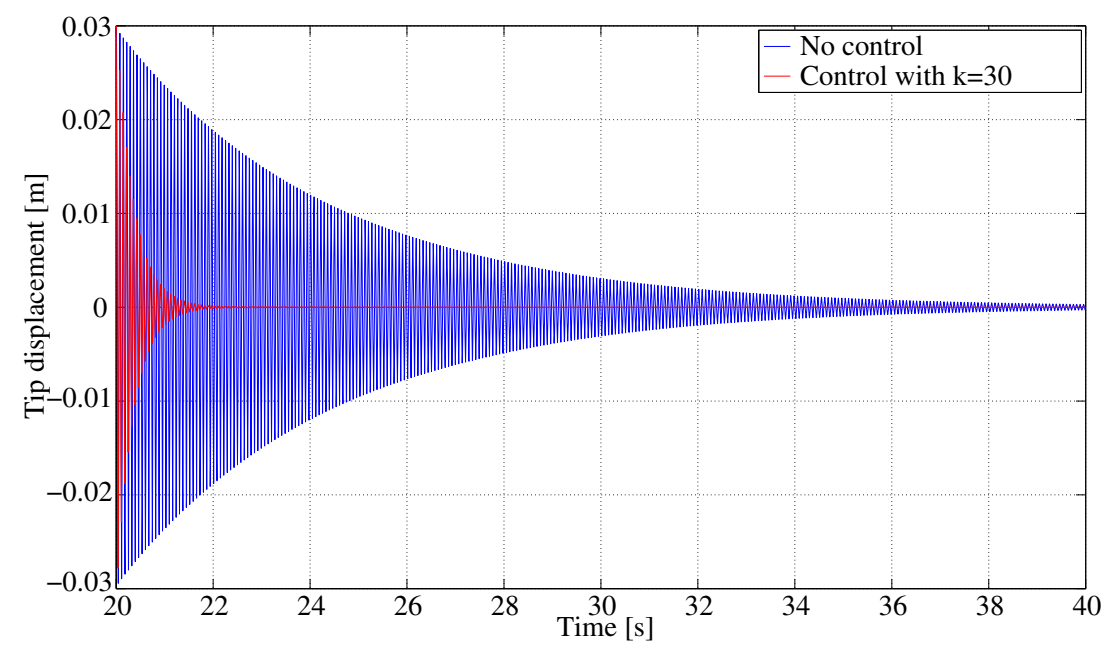

Figure 10: The Lyapunov stability theorem controller for $k=30$ (SE model).



Figure 11: The FFT spectrum of the smart beam using Lyapunov stability controller (SE model).

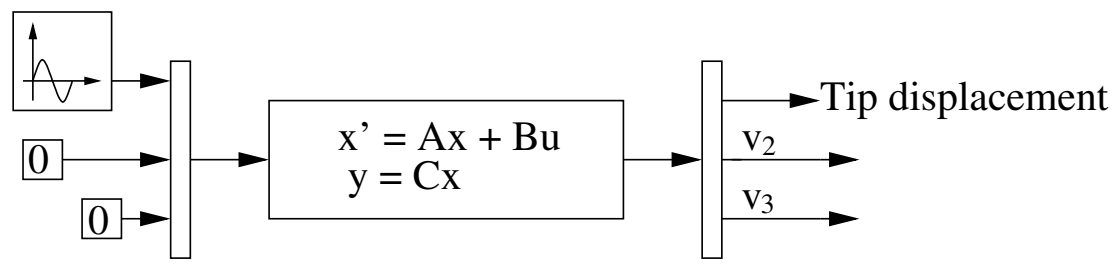

Figure 12: The SS model of the smart beam with controller (step 1).

The steps mentioned above could be also summarized in one step, but in this case a timer must be inserted in the model to deactivate the exciting force when vibrations become stable at $t=20 \mathrm{~s}$. The $S S$ representation of the smart beam in the second step is shown in Fig. 13.

A comparison of the results from the SE model and the SS model is shown in Fig. 14 and Fig. 15 where the time region between 20 and $22 s$ is magnified. It can be seen that both models yielded the same results. Nevertheless, much more time was needed to carry out the simulation in the 


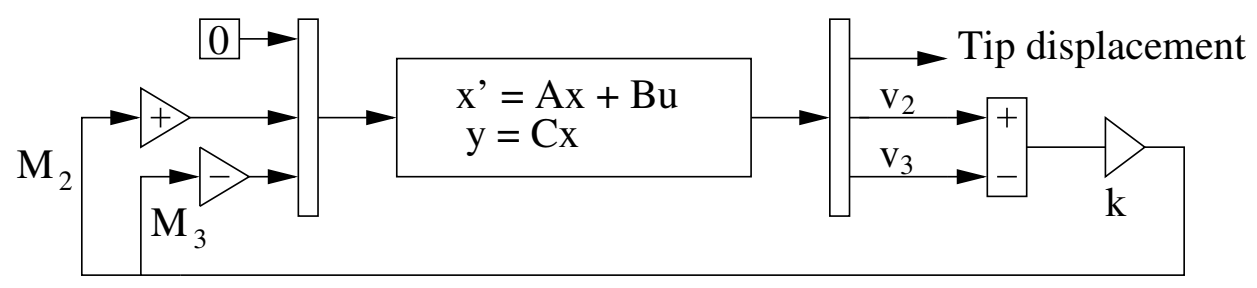

Figure 13: The SS model of the smart beam with controller (step 2).

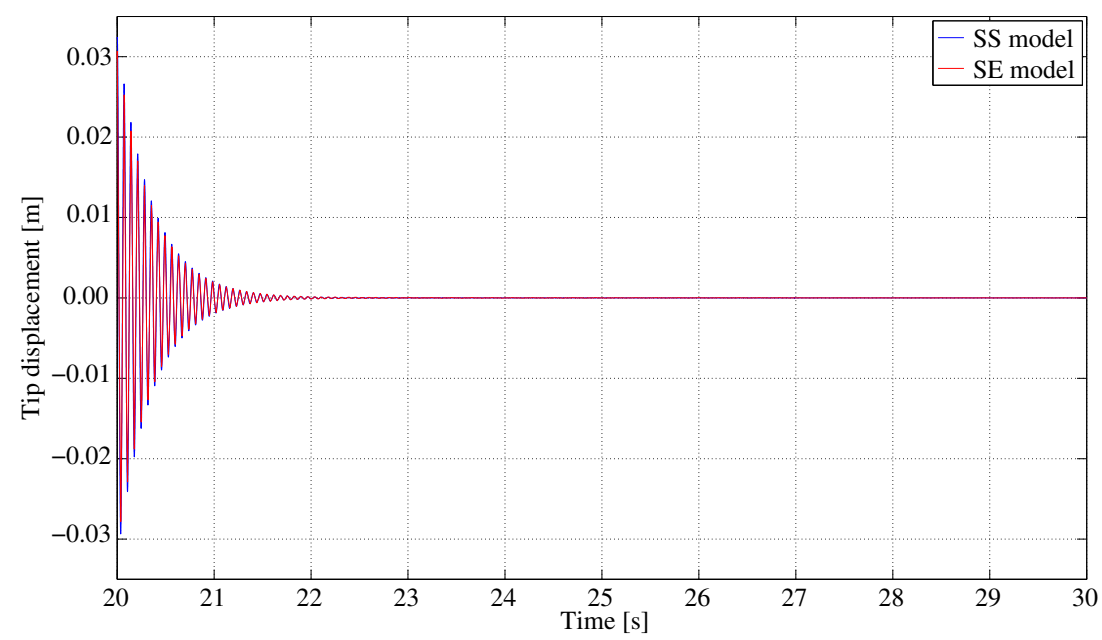

Figure 14: Tip displacement vs. time using SE and SS models.

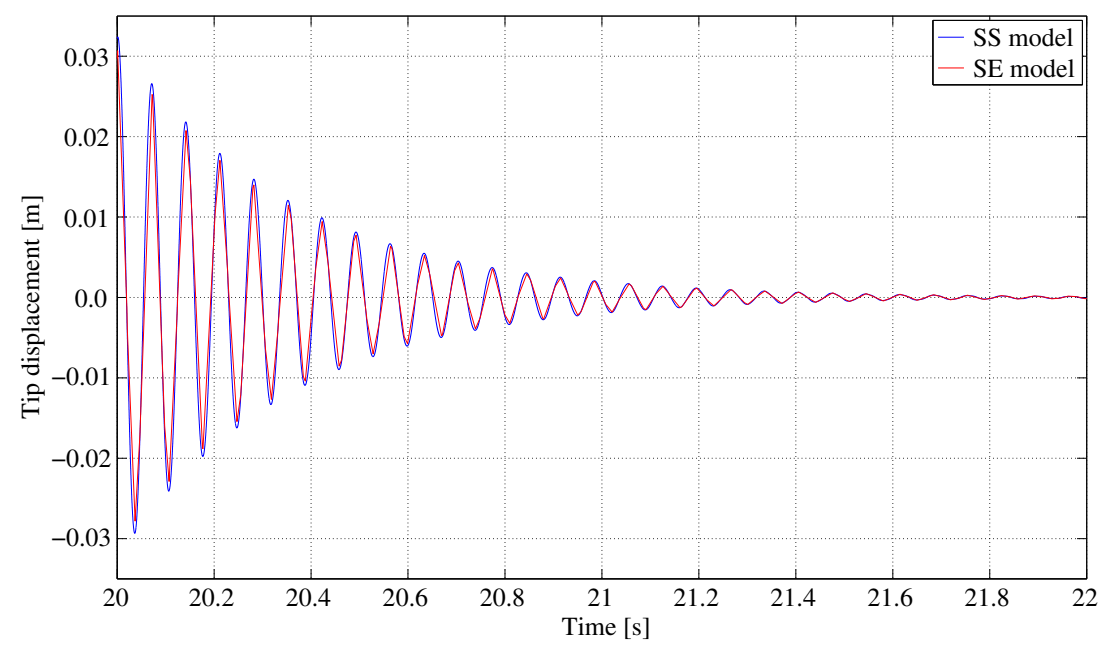

Figure 15: Tip displacement vs. time in a zoomed region of Fig. 14. 
SS model (about 35 minutes), while in the SE model, less time (only 3 minutes) was needed. This could be due to the fact that in the SE model a fixed time-step can be assigned (here $0.01 \mathrm{~s}$ ), while in the SS representation the time-step was automatically set. Moreover, the stresses and energy curves, could be requested in addition to the force vectors along the SE model. This is one of the advantages of the SE technique in comparison to the SS representation which is more practical and in which the controller can be easily implemented [33].

\section{SUMMARY}

In this work, a linear controller based on the Lyapunov stability theorem was designed and implemented on a reduced model of a smart structure. The state-space representation of the same model was extracted as well. The controller was implemented on this model too, and the results from both models were compared.

\section{REFERENCES}

[1] N. Ghareeb, Y. Radovcic, Fatigue analysis of wind turbines. DEWI magasin, 35, 12-16, 2009.

[2] R. Vepa, Dynamics of Smart Structures. John Wiley \& Sons, 2010.

[3] S. Narayanan, V. Balamurugan, Finite Element Modelling of Piezolaminated Smart Structures for Active Vibration Control with Distributed Sensors and Actuators. Journal of Sound and Vibration, 262, 529-562, 2003.

[4] A. Mallock, A Method of Preventing Vibration in Certain Classes of Steamships. Trans. Inst. Naval Architects, 47, 227-230, 1905.

[5] A. Vang, Vibration Dampening. U.S. Patent US 2,361,071, Oct. 24, 1944.

[6] M. Balas, Active Control of Flexible Systems. Journal of Optimization theory and Applications, 25, No. 3, 415-436, 1978.

[7] V. Piefort, Finite Element Modelling of Piezoelectric Active Structures. Universit Libre de Bruxelles, PhD Thesis, 2001.

[8] J. Fanson, J. Chen, Structural Control by the Use of Piezoelectric Active Members. Proceedings of NASA/DOD Control-Structures Interaction Conference, NASA CP-2447, 2 , 809-830, 1986.

[9] A. Preumont, Vibration Control of Active Structures, An Introduction. Kluwer Academic Publishers, 2002.

[10] S. Moheimani, A. Fleming, Piezoelectric Transducers for Vibration Control and Damping. Springer, 2006.

[11] K.B. Waghulde, B. Sinha, M. Patil, S. Mishra, Vibration Control of Cantilever Smart Beam by Using Piezoelectric Actuators and Sensors. International Journal of Engineering and Technology, 2, No. 4, 259-262, 2010. 
[12] A. Gani, A. Salami, R. Khan, Active Vibration Control of a Beam with Piezoelectric Patches: Real-time Implementation with xPC target. Proceedings of the IEEE Conference on Control Applications, CCA 2003, 1, 538-544, 23-25 June 2003.

[13] J. Block, T. Strganan, Applied Active Control for a Nonlinear Aeroelastic Structure. Journal of Guidance, Control, and Dynamics, 21, No. 6, 838-845, 1998.

[14] V.V. Varadan, Y. Lim, V.K. Varadan, Closed loop finite-element modeling of active/passive damping in structural vibration control. Smart Materials and Structures, 5, No. 5, 685694, 1996.

[15] U. Gabbert, H. Köppe, T. Trajkov, Entwurf intelligenter Strukturen unter Einbeziehung der Regelung (English: Design of intelligent Structures including Regulation). at Automatisierungstechnik 50, Oldenbourg Verlag, 9, 432-438, 2002.

[16] H. Allik, T. Hughes, Finite Element Method for Piezoelectric Vibration. International Journal for Numerical Methods in Engineering, 2, 151-157, 1970.

[17] N. Ghareeb, R. Schmidt, Modelling and control of a smart beam. PAMM Proc. Appl. Mech., 12, 703-704, 2012.

[18] N. Ghareeb, Design and Implementation of Linear Controllers for the Active Control of Reduced Models of Thin-Walled Structures. RWTH Aachen University, PhD Thesis, 2013.

[19] M. Petyt, Introduction to Finite Element Vibration Analysis. Cambridge University Press, 2003.

[20] R. Craig, M. Bampton, Coupling of Substructures for Dynamic Analyses. AIAA Journal, 6, No. 7, 1313-1319, 1968.

[21] C. Rickelt-Rolf, Modellreduktion und Substrukturtechnik zur effizienten Simulation dynamischer, teilgeschädigter Systeme (English: Model Reduction and Substructuring for Efficient Simulation of Dynamic Partially-Damaged Systems). Technische Universität Carolo-Wilhelmina zu Braunschweig, PhD Thesis, 2009.

[22] J. Butterworth, J. Lee, B. Davidson, Experimental Determination of Modal Damping From Full Scale Testing. 13th World Conference on Earthquake Engineering, Vancouver, Canada, August 1-6, 2004.

[23] A. Alipour, F. Zareian, Study Rayleigh Damping in Structures; Uncertainties and Treatments. The 14th World Conference on Earthquake Engineering, Beijing, China, October 12-17, 2008.

[24] L. Rayleigh, Theory of Sound (two volumes). Dover Publications, New York, 1877.

[25] S. Adhikari, J. Woodhouse, Identification of Damping: Part 1, Viscous Damping. Journal of Sound and Vibration, 243, No. 1, 43-61, 2001.

[26] I. Chowdhury, S. Dasgupta, Computation of Rayleigh Damping Coefficients for Large Systems. The Electronic Journal of Geotechnical Engineering, 8, Bundle 8C, 2001. 
[27] I. Giosan, Dynamic Analysis with Damping for Free-Standing Structures Using Mechanical Event Simulation. Autodesk Report, 2010.

[28] D. Ewins, Modal Testing: Theory and Practice. John Wiley \& Sons Inc., 1984.

[29] K. Ogata, Modern Control Engineering. Prentice Hall, 2002.

[30] H. Khalil, Nonlinear Systems. Prentice Hall, Madison, 1996.

[31] G. C. Gorain, S. K. Bose, Boundary stabilization of a hybrid Euler-Bernoulli beam. Proc. Indian Acad. Sci. (Math. Sci.), 1999.

[32] S.M. Newman, Active Damping Control of a Flexible Space Structure Using Piezoelectric Sensors And Actuators. Naval Postgraduate School, Master Thesis, 1992.

[33] N. Ghareeb, R. Schmidt, Modeling and Active Vibration Control of a Smart Structure. Proceedings of the 9th international Conference on Informatics in Control, Automation and Robotics (ICINCO 2012), 1, No. 86, 142-147, Rome, Italy, 2012

[34] O.C. Zienkiewicz, R.C. Taylor, The finite element method, Vol. I, 4th Edition. McGraw Hill, 1989.

[35] J.T. Oden, T. Belytschko, I. Babuska, T.J.R. Hughes, Research directions in computational mechanics. Computer Methods in Applied Mechanics and Engineering, 192, 913-922, 2003.

[36] J.H. Argyris, M. Papadrakakis, L. Karapitta, Elastoplastic analysis of shells with the triangular element TRIC. M. Papadrakakis, A. Samartin, E. Oñate eds. 4th International Colloquium on Computation of Shell and Spatial Structures (IASS-IACM 2000), Chania, Crete, Greece, June 4-7, 2000. 